07

\title{
Концентраторы солнечного излучения в паре с многопереходными фотоэлектрическими преобразователями в наземных гелиоэнергетических установках (часть I)
}

\author{
(C) Е.А. Ионова, ${ }^{1}$ М.В. Уланов, ${ }^{1}$ Н.Ю. Давидюк, ${ }^{2}$ Н.А. Садчиков ${ }^{1}$ \\ ${ }^{1}$ Физико-технический институт им. А.Ф. Иоффре РАН, \\ 194021 Санкт-Петербург, Россия \\ ${ }^{2}$ Санкт-Петербургский национальный исследовательский Академический университет РАН, \\ 194021 Санкт-Петербург, Россия \\ e-mail: ionova@mail.ioffe.ru
}

(Поступило в Редакцию 25 мая 2016 г.)

Разработан способ определения параметров концентратора излучения в гелиоэнергетических установках. Для оценки эффективности использования концентраторов в виде линз Френеля в установках с трехпереходными фотоэлектрическими преобразователями введено понятие эффективности пары „концентраторфотопреобразователь“. Предложен способ расчета преломляющего профиля концентраторов, учитывающий дисперсионную зависимость показателя преломления и ее изменение от температуры для материала преломляющего профиля концентратора-силиконового компаунда Wacker RT604. Результаты расчета позволяют добиться максимальной эффективности пары „концентратор-фотопреобразователь“ при наличии хроматических аберраций в оптической системе концентрирования солнечного излучения.

\section{Введение}

В мире постепенно растет внедрение гелиоэнергетических установок, в том числе на базе высокоэффективных (КПД $>40 \%)$ многокаскадных фотоэлектрических преобразователей (ФЭП) с несколькими $p-n$-переходами, изготовленными на основе гетероструктур в системе АЗВ5. Основной проблемой, возникающей при использовании таких высокоэффективных ФЭП, является их относительно высокая стоимость по сравнению с традиционными однопереходными ФЭП на основе кремния. Одним из преимуществ ФЭП на основе гетероструктур в системе АЗВ5 является возможность преобразования солнечного излучения (СИ) без потери эффективности при высоких уровнях концентрации света. Решением проблемы удешевления единицы мощности, вырабатываемой многокаскадными ФЭП, стало использование концентраторов солнечного излучения, перенаправляющих энергию СИ на миниатюрные ФЭП в фокусе концентратора. Таким образом, концентраторы обеспечивают экономию площади чипов ФЭП пропорционально кратности концентрирования $[1,2]$.

В работе рассматривается гелиоэнергетическая установка (ГЭУ), конструкция которой включает каркас, оснащенный приводами двухосевого слежения за положением Солнца, и установленные на нем фотоэлектрические модули [2,3]. Каждый модуль включает в себя от десятков до сотен идентичных пар концентраторФЭП. Функционально работа ГЭУ делится на слежение за положением Солнца на небесной сфере, концентрирование СИ и преобразование концентрированного СИ в фототок. Работа посвящена решению проблемы эффективного преобразования концентрированного излучения в фототок с учетом особенностей каскадной структуры ФЭП.

\section{Конструкция и оптические характеристики концентратора}

Концентратор выполнен в виде линзы Френеля квадратной формы из прозрачной силиконовой резины (CP) [2]. Концентратор как оптический элемент представляет собой набор концентрических конических поверхностей (ступеней), которые, если бы были соединены между собой, образовали бы асферическую плосковыпуклую линзу. Множество силиконовых концентраторов, составляющих концентраторную панель фотоэлектрического модуля, выполняются одновременно путем заливания в форму СР в жидком состоянии. В процессе перехода жидкой СР в резиноподобное состояние реплика концентраторов закрепляется на стеклянной пластине, которая нужна для обеспечения механической прочности и устойчивой формы концентраторной панели модуля. На другой панели, расположенной в фотоэлектрическом модуле параллельно концентраторной, расположены ФЭП таким образом, что геометрический центр их фоточувствительной области находится на оптической оси и в фокусе соответствующего концентратора.

В качестве материала для френелевского профиля концентратора используется прозрачная СР марки Elastosil RT604 фирмы Wacker [4]. Это двухкомпонентный силиконовый каучук, отличающийся хорошей текучестью в жидкой фазе и отсутствием усадки при переходе из жидкого в резиноподобное состояние, т.е. полученный профиль практически полностью совпадает с исходной формой.

В работе [3] показана спектральная зависимость пропускания образца, имитирующего концентратор, который представляет собой слой СР толщиной $0.5 \mathrm{~mm}$ на боросиликатном стекле толщиной $4 \mathrm{~mm}$. Образец 
почти полностью прозрачен в интервале длин волн $350-1200 \mathrm{~nm}$, а область поглощения в интервале длин волн 1650-1900 nm соответствует участку спектра СИ с низкой плотностью мощности. Моделирование концентратора СИ требует учета как пропускания, так и дисперсии показателя преломления СР. Дисперсия СР показана в работе [5] для интервала длин волн 300-1200 nm, но линию кривой можно продлить и до длины волны $1800 \mathrm{~nm}$ и не учитывать аномальную дисперсию вблизи полос поглощения 1650-1900 nm также по причине низкой плотности мощности СИ на этом участке.

Особенностью СР как оптического материала является значительное объемное тепловое расширение и соответствующее значительное изменение дисперсионной зависимости показателя преломления, что приводит к размытию светового пятна в фокусе концентратора.

С помощью рефрактометра ИРФ-22 были определены показатель преломления $n_{D} \mathrm{CP}$ марки Elastosil RT604 на длине волны $589 \mathrm{~nm}$ при $25^{\circ} \mathrm{C}$, а также зависимость $n_{D}$ от температуры. Исследование резиноподобного вещества потребовало внесения изменений в стандартную методику измерений ИРФ-22. На измерительную призму, предварительно обработанную адгезивным составом на основе силана, нанесли СР в жидком состоянии, в центре расположили измерительный контакт термопары и прижали слой осветительной призмой, создав между призмами тонкую пленку СР. После перехода СР в резиноподобное состояние производился нагрев призм. Одномоментные значения $n_{D}$ и $T$ были получены при естественном остывании призм. С учетом поправок, вводимых из-за изменения показателя преломления измерительной призмы при изменении температуры, были получены следующие результаты: при $T=0-100^{\circ} \mathrm{C}$

$$
n_{D}(T)=1.4147-3.7781 \cdot 10^{-4} T \text {. }
$$

Таким образом, при $T=25^{\circ} \mathrm{C}$

$$
n_{D}^{25}=1.4052
$$

Дисперсию СР $n(\lambda)$, представленную в работе [5], можно отождествить с функцией

$$
n(\lambda)=a_{1} \exp \left(-\lambda / t_{1}\right)+a_{2} \exp \left(-\lambda / t_{2}\right)+a_{3},
$$

где $a_{1}, a_{2}, t_{1}, t_{2}$ равны соответственно 0.72, 0.04, 94.0, 490.0.

Для интервала температур $0-50^{\circ} \mathrm{C}$ принимаем, что от температуры будет зависеть только сдвиг дисперсионной кривой вдоль ординаты, определяемый параметром $a_{3}$, но не форма кривой. Тогда для температуры $25^{\circ} \mathrm{C}$ и длины волны $589 \mathrm{~nm}$, исходя из (2), $a_{3}$ равен 1.3919 (рис. $\left.1, a\right)$.

По формулам (1) и (3) можно определить ход дисперсионной кривой CP марки Elastosil RT604 для любой температуры, для которой можно применить приближение вертикального сдвига.

\section{Преобразование СИ со спектром AM1.5D многопереходными ФЭП}

В составе ГЭУ используются ФЭП, произведенные методом МОС-гидридной эпитаксии, представляющие собой гетероструктуру с тремя активными $p-n$-переходами. Они предназначены для преобразования СИ: области поглощения их активных $p-n$-переходов (далее переходов) с близким к 100\% внутренним квантовым выходом последовательно перекрывают спектр СИ в широком интервале 350-1800 nm. Спектр поглощения данных ФЭП не изменяется при увеличении плотности мощности поглощенного излучения в несколько тысяч раз больше стандартных $1000 \mathrm{~W} / \mathrm{m}^{2}$. Это свойство открыло перспективы для ГЭУ с двухосевым слежением за Солнцем и концентрированием СИ.

В связи с суточным и годовым движением Земли и в связи с атмосферными условиями изменяются как плотность мощности, так и спектр СИ. Общепринято настраивать максимум по выходным характеристикам наземных солнечных элементов на спектр AM1.5D (прямое излучение). Принципиальная особенность многопереходных ФЭП состоит в том, что, поглощая излучение, каждый переход генерирует свой уровень фототока, но общий фототок ФЭП будет тождествен наименьшему из них. То есть переходы соответствуют последовательно соединенным источникам тока, согласно аналоговой модели функционирования ФЭП. Поэтому в случае освещения их фоточувствительной области СИ при зенитальном положении Солнца, когда усилена коротковолновая часть спектра, уровень фототока ФЭП не увеличится. Но зато при освещении ФЭП СИ со спектрами, приближающимися к рассветным или закатным, когда коротковолновая часть значительно ослабляется, уровень фототока будет падать слабее, чем если бы ФЭП был настроен на зенитальный спектр.

В настоящей работе расчет и исследование пар концентратор-ФЭП проведены для спектра AM1.5D при плотности мощности СИ 1000, падающего на концентратор, $1000 \mathrm{~W} / \mathrm{m}^{2}$.

\section{Эффективность пары концентратор-ФЭП}

Эффективность пары (ЭП) - основная характеристика пары концентратор-ФЭП, определяющая фототок ГЭУ. ЭП вычисляется по достаточно объемному алгоритму, так как фототок ГЭУ сложным образом зависит от спектрального состава СИ, оптических свойств концентратора, характеристик ФЭП, конструкции концентратора.

Описание алгоритма вычисления начнем с оптической схемы концентрирования СИ. СИ, падающее под прямым углом к концентратору, взаимодействует с рассматриваемой парой, сначала проходя сквозь плоскую 

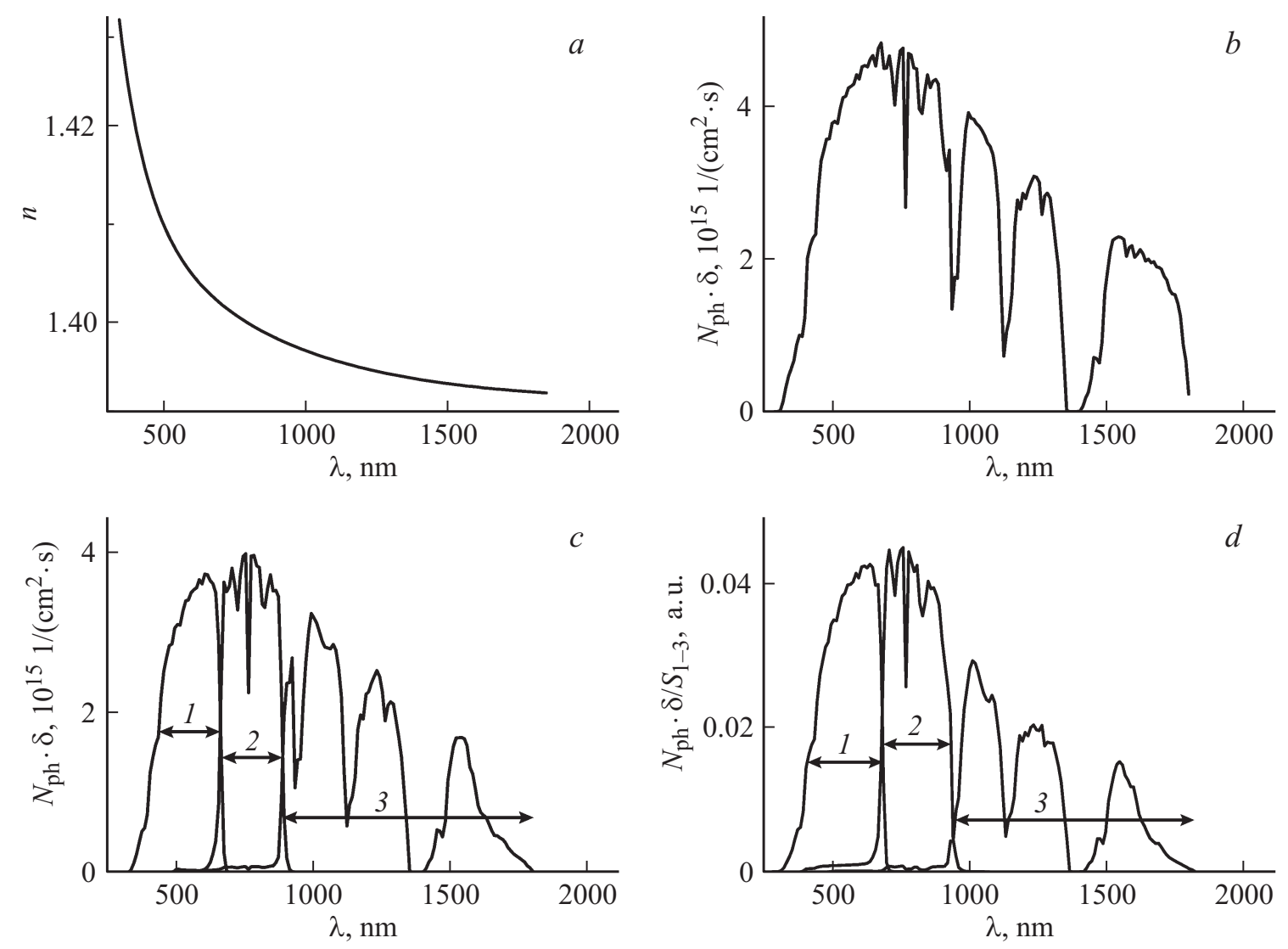

Рис. 1. Дисперсия показателя преломления $n$ силиконовой резины Wacker RT 604 при температуре $25^{\circ} \mathrm{C}(a)$; спектр солнечного излучения AM1.5D с плотностью мощности $1000 \mathrm{~W} / \mathrm{m}^{2}(b)$; мультиплицированные спектры, полученные умножением спектра $(b)$ на спектральные характеристики $p-n$-переходов (участки $1-3$ ) фотоэлектрического преобразователя, обозначенного ФЭП № 1 (c); нормированные мультиплицированные спектры, полученные умножением спектра $(b)$ на спектральные характеристики другого фотоэлектрического преобразователя, обозначенного ФЭП № 2, и разделенные на сумму своих элементов $(d)$.

стеклянную боросиликатную пластину толщиной $4 \mathrm{~mm}$, затем сквозь прикрепленную к ее внутренней стороне линзу с френелевской поверхностью из прозрачной СР толщиной до $0.5 \mathrm{~mm}$. При выходе из линзы обратно в воздушную среду ход лучей преломляется и они пересекаются. Центр фоточувствительной области ФЭП расположен точно на оптической оси концентратора в зоне пересечения преломленных лучей СИ, а его плоскость параллельна плоскости стеклянной пластины (рис. 2).

В отличие от однопереходного ФЭП, в котором генерация тока прямо пропорциональна числу фотонов, попавших на фоточувствительную область, в случае многопереходного ФЭП уровень фототока зависит еще и от спектрального состава СИ. А именно, если три перехода при освещении неконцентрированным СИ со спектром AM1.5D и плотностью мощности $1000 \mathrm{~W} / \mathrm{m}^{2}$ выдают фототоки $J_{1}, J_{2}, J_{3}$, то фототок, выдаваемый ФЭП, определяется как $\min \left(J_{1}, J_{2}, J_{3}\right)$. При освещении таким же, но концентрированным в $N$ раз СИ фототок, выдаваемый ФЭП, равен $\min \left(N J_{1}, N J_{2}, N J_{3}\right)$, но только в случае попадания $100 \%$ фотонов на фоточувствительную область ФЭП. На практике включение концентратора в систему СИ-преобразователь, во-первых, уменьшает передаваемую мощность СИ на несколько процентов из-за оптических потерь, во-вторых, получающаяся значительная экономия чипов ФЭП может компенсировать разработчикам конструкции ГЭУ потерю еще нескольких процентов передаваемой мощности СИ из-за вышедших за пределы фоточувствительной области ФЭП фотонов.

Расчет ЭП концентратор-ФЭП должен проводиться не для целого спектра СИ AM1.5D, а для новых спектров на его основе и на основе спектральных характеристик $p-n$-переходов ФЭП. Спектр AM1.5D представили в единицах фотонов $N_{\mathrm{ph}}$ с энергиями в условном интервале длин волн $\delta=10 \mathrm{~nm}$, пересекающих в секунду площадь в $1 \mathrm{~cm}^{2}$ (рис. $\left.1, b\right)$. При умножении этого спектра и спектров внешнего квантового выхода $p-n$-переходов, представляемых в процентах, получили мультиплицированные спектры (MC) для каждого перехода из трех - $\mathrm{MC}_{1}, \mathrm{MC}_{2}, \mathrm{MC}_{3}$ (рис. $1, c$ ). $\mathrm{MC}_{1-3}$ - это спектры „полезного“ СИ, вносящего вклад в фототоки $p-n$-переходов. Абсолютное число фотонов для вычисления ЭП значения не имеет, поэтому эти спектры используются в нормированном виде (рис. $1, d$ ). Все элементы спектра $\mathrm{MC}_{i}$ для перехода $i=1-3$ после 


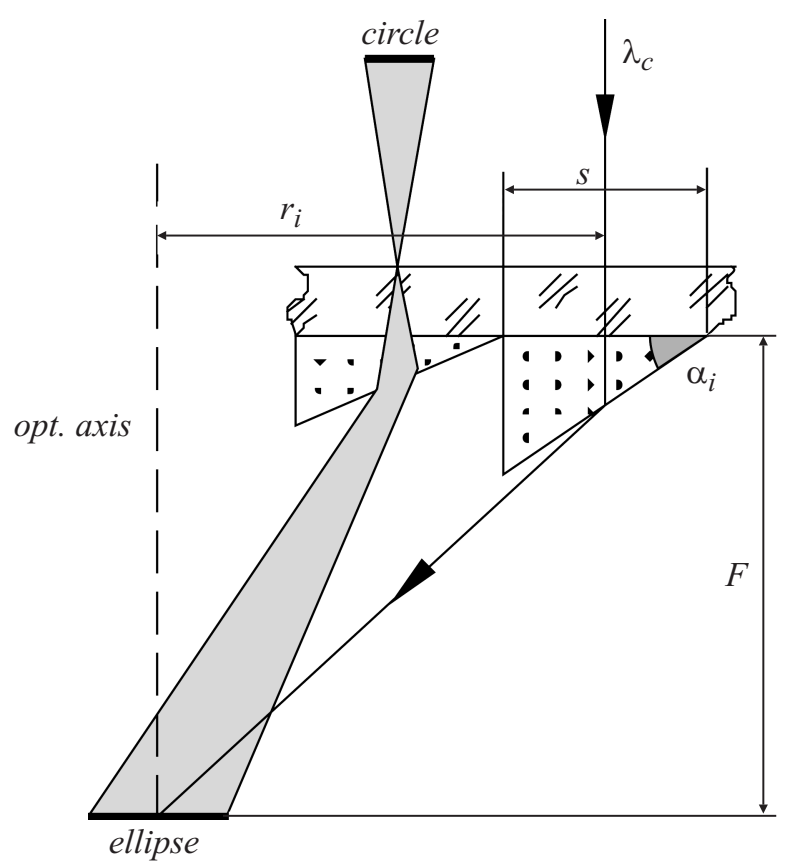

Рис. 2. Параметры концентратора: $F-$ фокусное расстояние, $r_{i}$ - расстояние от центра $i$-й преломляющей поверхности до оптической оси, $s-$ ширина преломляющей поверхности, $\alpha_{i}$ - угол наклона $i$-й преломляющей поверхности. Условие для формулы (5): луч с длиной волны $\lambda_{c}$ падает перпендикулярно на середину преломляющей поверхности и после преломления пересекает точку фокуса (справа). Схематическое представление одного из множества сходящихся пучков солнечных лучей на основании концентратора и его проекции на фокусную плоскость после преломления (слева).

деления на сумму $S_{i}$ элементов спектра $\mathrm{MC}_{i}$ в сумме дают единицу, имеющую смысл „одного солнца“, только с той частью СИ, которая может преобразоваться $p-n$-переходом $i$.

В связи с трехпереходной структурой ФЭП в алгоритме вычисления ЭП имеются три однотипных процедуры - это вычисление для каждого перехода $i=1-3$ доли $\eta_{i}$ прошедшего через концентратор и попавшего на фоточувствительную область ФЭП излучения из $\mathrm{MC}_{i}$. Вычисление проводилось с помощью специально разработанной программы на $\mathrm{C}++$ отдельно для последовательных интервалов длин волн шириной $\delta$ и средних значений оптических характеристик материалов концентратора на этих интервалах.

В алгоритме вычисления СИ в виде спектра $\mathrm{MC}_{i}$, исходя из положений теоретической фотометрии, представляется в виде совокупности равномерно распределенных по концентратору сходящихся пучков геометрических лучей. Каждый пучок включает лучи, исходящие из точек излучающей поверхности - диска Солнца и сходящиеся в одной из точек поверхности концентратора (рис. 2). Для любого интервала длин волн шириной $\delta$, например под номером $j$, соответствующая доля фотонов из $\mathrm{MC}_{i}$ принималась распределенной поровну внутри совокупности монохромных пучков лучей c длиной волны $\lambda_{j}$ в середине данного интервала $j$. (В работе не учитывается спектральное распределение в пучке лучей, идущих от Солнца - краевое затемнение.)

Преломление пучка на одной из конических поверхностей концентратора приводит к изменению формы проекции пучка на фокусной плоскости. Проекция в форме круга превращается в фигуру, в физическом приближении близкую к эллипсу. Таким образом, энергия пучка, за вычетом оптических потерь, переносится на определенную плоскость под концентратором в область, ограниченную эллипсом. Уравнение эллипса определялось по координатам пересечения этой плоскости тремя лучами пучка - центрального и двух крайних, относящихся к осям эллипса. Ход этих лучей через концентратор и в воздушном пространстве за ним определялся методами аналитической геометрии.

Для оценки энергетического вклада пучка учитывались потери, определенные отражениями СИ от внешней стороны стеклянной пластины, от перехода из стекла в СР и от границы СР-воздух. Энергетические вклады всех преломившихся пучков с длиной волны $\lambda_{j}$, оставаясь выраженными в долях от общего числа фотонов, суммировались на координатной сетке, покрывающей фокусную плоскость. Затем эта и аналогичные суммы, определенные для совокупности монохроматических пучков с длинами волн в середине остальных интервалов шириной $\delta$, суммировались между собой также на координатной сетке. Так рассчитывалось распределение в фокусном пятне фотонов из $\mathrm{MC}_{i}$. Доля $\eta_{i}$ попавших на фоточувствительную область ФЭП фотонов определялась суммированием вкладов ячеек координатной сетки внутри границы фоточувствительной области ФЭП. (Ширина интервала $\delta$, число сходящихся пучков лучей на концентраторе, размер ячейки координатной сетки выбирались так, чтобы их величины не влияли на результат.)

Плотности фототока $J_{1-3}$-переходов, определенные в условиях неконцентрированного СИ спектра AM1.5D, и плотности мощности $1000 \mathrm{~W} / \mathrm{m}^{2}$ сохраняют свое значение при освещении фотонами из $\mathrm{MC}_{1-3}$ из-за тождественности этих спектров в аспекте поглощения ФЭП. Для определения ЭП имеет значение только соотношение параметров $J$. Величины $k_{1}=J_{1} / J_{\min }, k_{2}=J_{2} / J_{\min }$, $k_{3}=J_{3} / J_{\min }$, где индекс min соответствует наименьшему из трех фототоков, - это увеличивающие коэффициенты для долей $\eta_{1-3}$, прошедшего через концентратор и попавшего на фоточувствительную область ФЭП излучения из $\mathrm{MC}_{1-3}$. Например, выход половины фотонов спектра $\mathrm{MC}_{i}$ после концентрирования за пределы ФЭП компенсируется тем, что $p-n$-переход $i$ выдает в два раза больший фототок на „одном солнце“ $\left(k_{i}=2\right)$. Эффективность пары концентратор-ФЭП $\eta$ определяется следующим выражением:

$$
\eta=\min \left(\eta_{1} k_{1}, \quad \eta_{2} k_{2}, \eta_{3} k_{3}\right) \cdot 100 \%
$$

В первой части работы исследуются пары концентратор-ФЭП, в которых ФЭП, обозначенный ФЭП № 1, 
выполнен на основе гетероструктуры GaInP/GaAs/Ge, имеет размеры $2 \times 2 \mathrm{~mm}$ и фоточувствительную область круглой формы с диаметром $1.7 \mathrm{~mm}$. Его внешний квантовый выход, умноженный на спектр Солнца, представлен в спектрах $\mathrm{MC}_{1-3}$ на рис $1, c$. Плотности фототока для трех переходов $J_{1-3}=13.14,13.29,20.36 \mathrm{~mA} / \mathrm{cm}^{2}$, они соответствуют коэффициентам $k_{1-3}=1,1.01,1.55$.

\section{Решение концентратора СИ для ГЭУ}

Качество концентрирования определяет совокупность концентрических конических поверхностей линзы Френеля: их углы наклона и размер. Углы наклона ступеней френелевской поверхности в настоящей работе рассчитываются из условия, что при температуре концентратора $T_{c}$ луч $\mathrm{c}$ длиной волны $\lambda_{c}$, падающий на среднюю точку ступени концентратора параллельно оптической оси, после преломления отсечет в точке пересечения с оптической осью отрезок, равный фокусному расстоянию $F$ (рис. 2). Угол наклона $i$-й ступени шириной $s$, центр которой отстоит от оптической оси на расстоянии $r_{i}=s i-s / 2$, определяет функция $\alpha_{i}$, представленная в виде уравнения (5).

$$
\operatorname{arctg} \frac{2 r_{i}}{2 F-s \operatorname{tg} \alpha_{i}}=\arcsin \left(n_{c} \sin \alpha_{i}\right) .
$$

Параметр $n_{c}-$ расчетный показатель преломления $\mathrm{CP}$, соответствующий в дисперсионной зависимости для заданной температуры $T_{c}$ длине волны $\lambda_{c}$ указанного луча.

В рамках рассматриваемой концепции ГЭУ интерес представляют пары концентратор-ФЭП, показывающие наибольшую ЭП при наименьшем расходе чипов ФЭП. Расход чипов ФЭП и ЭП определяется размытием светового пятна в фокусе концентратора. Имеются четыре причины его размытия, которые связаны с природой СИ, с конструкцией и материалом концентратора. Во-первых, из-за дисперсии показателя преломления силикона лучи с меньшей, чем $\lambda_{c}$, длиной волны отсекут на оптической оси отрезок меньше фокусного расстояния $F$, а лучи с большей длиной волны - отрезок больше $F$. При этом в фокальной плоскости там, где должна располагаться фоточувствительная область ФЭП, будет расширение светового пятна и его спектральное расслоение. Этот вклад в размытие светового пятна можно минимизировать, правильно подобрав для расчета концентратора $\lambda_{c}$ луча и соответственно параметр $n_{c}$.

Во-вторых, световое пятно в фокусе расширяется примерно на одну сотую фокусного расстояния из-за расхождения падающих на концентратор солнечных лучей в пределах $\pm 16^{\prime}$. В-третьих, световое пятно уширяется за счет изменения в фокальной плоскости соотношения полуосей эллипса в сторону „растягивания“, возрастающее для пучков геометрических лучей от центра к краю (углу) концентратора в зависимости от соотношения фокуса и размера концентратора. Дополнительно имеет

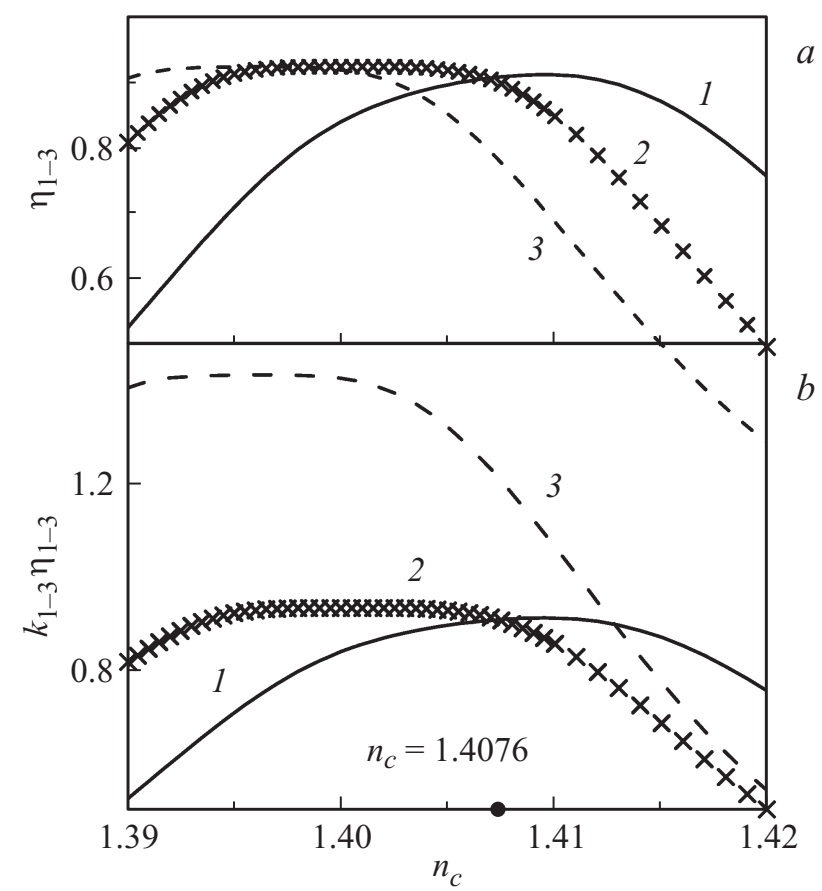

Pис. 3. (a) Число фотонов $\eta_{1-3}$, прошедших концентратор и попавших на фоточувствительную область ФЭП № 1, в долях от падающих на концентратор фотонов из $\mathrm{MC}_{1-3}$ в зависимости от параметра расчета концентратора $n_{c}$ (кривые 1-3 для трех $p-n$-переходов). (b) Зависимости из части $(a)$, умноженные на коэффициенты $k_{1-3}$ : для первого $p-n$-перехода $k_{1}=1.0$, для второго $k_{2}=1.01$, для третьего $k_{3}=1.55$.

место размытие светового пятна на ширину ступени $s$ из-за формы ступеней френелевской поверхности, не имеющих подфокусирующего изгиба. Поэтому решением стали концентраторы с маленькими ступенями френелевского профиля равной ширины.

Обобщая, расход чипов ФЭП и ЭП зависит и от абсолютных значений параметров концентратора и ФЭП, и от множества их взаимных соотношений.

Фокусное расстояние $F$, размер ступени $s$, размер концентратора $a$ - параметры, относящиеся к конструкции концентратора, фотоэлектрического модуля и ГЭУ. Выбор их значений связан не только с требованием высокой ЭП, но и с условиями в области экономической эффективности и технического воплощения. От параметра $n_{c}$, напротив, зависит только ЭП. В работе предлагается для пары концентратор-ФЭП с известным набором относящихся к конструкции параметров с помощью вычислительного алгоритма подбирать $n_{c}$, обеспечивающий наибольшую ЭП. Затем вычислительными методами предлагается исследовать свойства пары концентратор-ФЭП с известным набором параметров и оптимальным расчетным показателем преломления $n_{c}$, чтобы по результатам оценить целесообразность воплощения данной пары концентратор-ФЭП с известными параметрами в реальную конструкцию.

Из рассчитанных с помощью вычислительного алгоритма распределений фотонов из $\mathrm{MC}_{1-3}$ в фокальной 


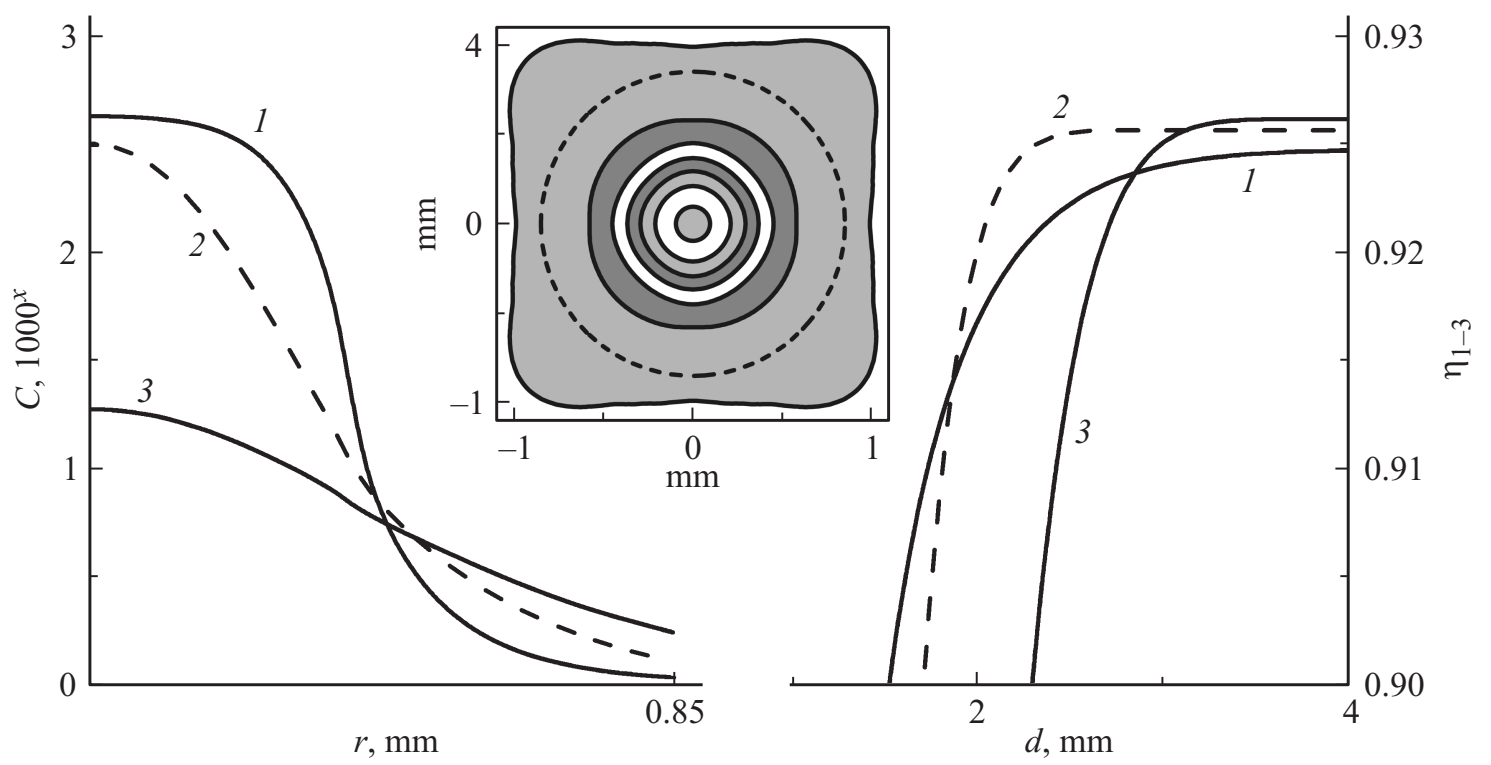

Рис. 4. Слева: зависимости величины локальной концентрации $C$ солнечного излучения от расстояния до оптической оси вдоль радиуса ФЭП, параллельного диагонали концентратора, построенные для трех $p-n$-переходов (линии $1,2,3)$. Справа: число фотонов $\eta_{1-3}$, прошедших концентратор и попавших на ФЭП № 1 с условно изменяющимся диаметром, в долях от падающих на концентратор фотонов из $\mathrm{MC}_{1-3}$, в зависимости от величины диаметра ФЭП № 1. Вставка: схема светового пятна для второго $p-n$-перехода ФЭП № 1, где первая смена заливки показывает изменение локальной концентрации от 1 до $400^{x}$, последующие смены заливки показывают увеличение локальной концентрации на $400^{x}$; диаметр $1.7 \mathrm{~mm}$ фоточувствительной поверхности ФЭП № 1 показан штрихами.

(или параллельной ей) плоскости определяются распределения локальной концентрации (ЛК) СИ в виде $\mathrm{MC}_{1-3}$. ЛК - безразмерная величина, показывающая, во сколько раз больше попало фотонов на ячейку координатной сетки в световом пятне по сравнению с числом фотонов на этом участке при отсутствии концентрации.

При некоторых предельных значениях ЛК СИ в фокусе концентратора, меняющихся от партии к партии произведенных ФЭП, происходит резкое уменьшение вырабатываемой мощности. В связи с этим при исследовании пар концентратор-ФЭП наряду с другими характеристиками указываются максимальные ЛК в трех распределениях.

\section{Концентратор для ФЭП № 1}

Расчет концентратора для ФЭП № 1 приведен для заданного набора параметров: фокус $F=80 \mathrm{~mm}$, сторона квадратного концентратора $a=40 \mathrm{~mm}$, ступени профиля Френеля шириной $0.25 \mathrm{~mm}$, температура, при которой произошла сшивка полимерной матрицы, и базовая температура исследования $T_{c}=25^{\circ} \mathrm{C}$. Минимальный размер светового пятна в фокусе этого концентратора составляет $0.01 F+s=1.05 \mathrm{~mm}$, поэтому пара с ФЭП с диаметром фоточувствительной области $1.7 \mathrm{~mm}$ обеспечена запасом площади, необходимым из-за расширения светового пятна.

Вначале для концентраторов, рассчитанных по формуле (5) для последовательности значений параметра $n_{c}$, был произведен расчет долей $\eta_{1-3}$ прошедшего через концентратор и попавшего на фоточувствительную область ФЭП № 1 излучения из $\mathrm{MC}_{1-3}$. Результаты приведены на графике (рис. 3,a). Видно, что имеются „предпочтительные“ для каждого перехода интервалы значений $n_{c}$, обусловленные каскадной структурой ФЭП и дисперсией показателя преломления СР. Для первого перехода это интервал $1.406-1.412$, для второго 1.395-1.406, для третьего 1.390-1.402. Расчетный показатель преломления $n_{c}$, оптимальный для данной пары концентратор-ФЭП № 1, определялся по графику, на котором рассчитанные зависимости умножены на соответствующие коэффициенты $k$ (кривые $1,2,3$ на рис. $3, b)$. Ход ЭП $\eta$ совпадает с ходом самой низкой по шкале кривой: сначала это кривая 1, относящаяся к первому переходу, после пересечения - кривая 2, относящаяся ко второму. Точка пересечения кривых 1 и 2 соответствует максимальной ЭП $\eta$ и соответственно оптимальному значению $n_{c}=1.4076$ (точка на оси). Зависимость ЭП $\eta$ от параметра $n_{c}$ с ростом отклонения от оптимального $n_{c}$ показывает резкое убывание.

Отметим, что ход кривой 3 показывает избыточную концентрацию генерированных носителей третьим $p-n$-переходом.

Были вычислены распределения фотонов из $\mathrm{MC}_{1-3}$ в фокусе пары концентратор-ФЭП № 1 с известными параметрами и оптимальным $n_{c}$ и из них рассчитаны зависимости, представленные на рис. $4, a, b$. На правой части рисунка показаны зависимости для трех $p-n$-переходов величины доли $\eta_{1-3}$ попавших на фоточувствительную 


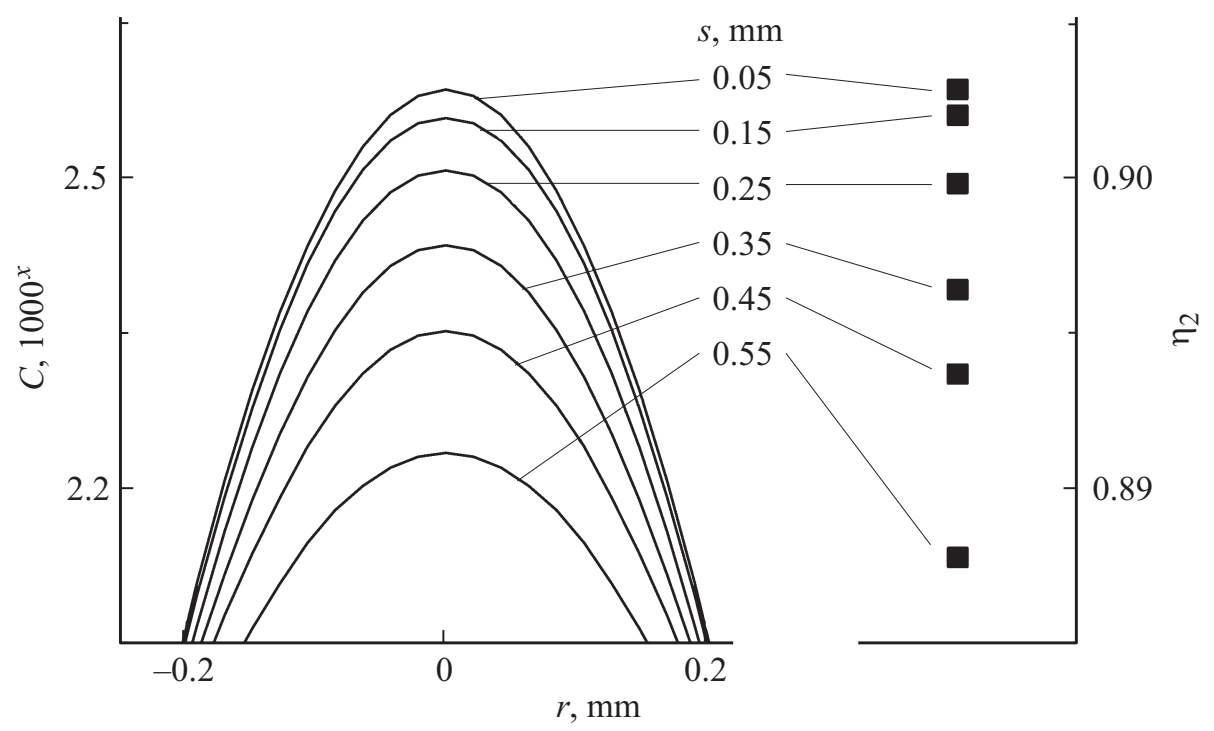

Рис. 5. Слева: зависимости величины локальной концентрации $C$ солнечного излучения в виде спектра $\mathrm{MC}_{2}$ для второго $p-n$-перехода ФЭП № 1 от расстояния до оптической оси вдоль параллельных диагонали концентратора радиусов $r$, построенные для концентраторов с разной шириной преломляющих поверхностей $s$. Справа: число фотонов $\eta_{2}$, прошедших концентратор и попавших на ФЭП № 1, в долях от падающих на концентратор фотонов из $\mathrm{MC}_{2}$, вычисленное для концентраторов с $s=0.05-0.55 \mathrm{~mm}$.

область фотонов по мере условного увеличения диаметра ФЭП № 1. Представление результатов расчета в такой форме позволяет исследовать концентрирование СИ безотносительно к диаметру конкретного ФЭП.

Полный размер частей светового пятна, относящихся к трем переходам, составляют 4.7/2.7/3.8 mm. Причиной наибольшего размытия светового пятна для первого перехода является широкий диапазон изменения показателя преломления на соответствующем спектральном участке дисперсионной зависимости. В то же время в центральной области светового пятна для первого перехода сосредоточено больше всего фотонов: например, $\eta_{1-3}$ равна 0.9 на внутренних областях световых пятен диаметрами $1.5 / 1.7 / 2.3 \mathrm{~mm}$. В случае предельно большого ФЭП $\eta_{1-3}$ составила бы 0.9248/0.9257/0.9263 для трех переходов. Отклонение $\eta_{1-3}$ от единицы обусловлено отражением от внешних поверхностей концентратора (стекло и СР).

Для диаметра ФЭП $1.7 \mathrm{~mm} \eta_{1-3}$ для трех переходов составила 0.9093/0.9002/0.7831, а ЭП $\eta$ после умножения $\eta_{1-3}$ на соответствующие коэффициенты $k$ составит $90.92 \%$ (4).

На рис. 4, $a$ представлены распределения ЛК СИ в виде $\mathrm{MC}_{1-3}$ для рассматриваемой пары концентраторФЭП № 1 в сечении плоскостью, проходящей через диагональ концентратора параллельно оптической оси. Из-за симметрии показаны только половины сечений от центра до края ФЭП. На графике наглядно показано несоответствие распределений ЛК для трех переходов. Уже отмеченная наибольшая собранность на уровне 0.9 светового пятна для первого перехода отразилась и на форме соответствующего распределения ЛК. Распределение для первого $p-n$-перехода имеет плоскую вершину радиуса $0.33 \mathrm{~mm}$ с уровнем концентрации больше $2000^{x}$, кольцо с резким спадом ЛК до $500^{x}$ и радиуса $0.47 \mathrm{~mm}$ и кольцо с плавным спадом до $62^{x}$ и радиуса $0.75 \mathrm{~mm}$. Последняя часть этого распределения - длинный и тонкий хвост, на который приходится доля фотонов 0.026. Распределения ЛК для двух других переходов отличаются по форме, так как не имеют плоской вершины, и по величине максимумов ЛК. Рассматриваемая пара концентраторФЭП № 1 показывает следующие максимальные значения ЛК: $2634^{x} / 2508^{x} / 1276^{x}$. Пологое распределение ЛК для третьего перехода с малым максимумом в итоге даст избыток генерированных носителей тока из-за увеличивающего коэффициента $k_{3}=1.55$.

На вставке к рис. 4 показана двумерная схема светового пятна для второго перехода исследуемой пары, на которой заливкой обозначена ЛК солнечного излучения в виде $\mathrm{MC}_{2}$, разделенная на уровни. Каждому уровню соответствует изменение ЛК на $400^{x}$. На первом уровне при изменении ЛК от $1^{x}$ до $400^{x}$ происходит переход от квадратного сечения к круглому. Первый уровень имеет большое размытие к квадрату из-за квадратной формы концентратора, однако в зонах пятна, относящихся к его углам, плотность фотонов низкая. За штриховую линию, обозначающую диафрагму диаметром $1.7 \mathrm{~mm}$, попало $1.65 \%$ фотонов спектра $\mathrm{MC}_{2}$. Следующие сечения уже имеют форму, близкую к круглой.

В рамках исследования актуальна оценка влияния ширины $s$ ступени профиля Френеля на эффективность пары концентратор-ФЭП. Она проведена на примере второго $p-n$-перехода ФЭП № 1 , как дающего наиболее „собранное“ световое пятно в фокусе, в паре с концентраторами с известными $F=80 \mathrm{~mm}$ и $a=40 \mathrm{~mm}$, но 
варьируемой $s$. Для каждого концентратора с набором параметров $F, a, s$ определялся оптимальный $n_{c}$. Для этого $n_{c}$ вычислялось распределение ЛК солнечного излучения спектра $\mathrm{MC}_{2}$ в фокальной плоскости, доля попавших на фоточувствительную область фотонов $\eta_{2}$.

На рис. 5 показаны верхние части распределений ЛК в том же сечении, что и для рис. 4, демонстрирующие собой увеличивающееся размытие светового пятна с ростом $s$. На правой части рисунка приведены значения $\eta_{2}$ вдоль оси ординат, относящиеся к приведенной на рисунке последовательности значений $s$ в интервале 0.05-0.55 mm. С последовательным ростом $s$ наблюдается ускоренное уменьшение $\eta_{2} \cdot 100 \%$ от 90.3 до $88.8 \%$.

\section{Заключение}

Данная работа представляет собой первую часть исследования концентратора солнечного излучения для многопереходного фотоэлектрического преобразователя. Исследование включает расчет углов наклона преломляющих поверхностей концентратора и выявление влияния параметров концентратора и параметров ФЭП на свойства пары.

В первой части исследования определены основные критерии оценки характеристик пары концентраторФЭП, а именно: введены понятия эффективности пары (ЭП) $\eta$ и распределения ЛК по фоточувствительной поверхности ФЭП; описан специально разработанный вычислительный алгоритм на $\mathrm{C}++$ для расчета этих характеристик. В отдельных пунктах приведены результаты исследования оптических свойств силиконовой резины и обоснование использования в вычислительном алгоритме спектра солнечного излучения AM1.5D для расчета и определения свойств концентраторов.

Оптимальный набор углов наклона $\alpha$ преломляющих поверхностей концентратора предложено определять для заданных фокусного расстояния $F$, стороны концентратора $a$, ширины ступени профиля Френеля $s$ и оптимального расчетного показателя преломления $n_{c}$. Оптимальный $n_{c}$ предложено определять методом подбора, вычисляя для каждого эффективность пары и выбирая наибольшую и ей соответствующий $n_{c}$.

Проведен частичный анализ свойств конкретной пары концентратор-ФЭП № 1. Параметры концентратора $F=80 \mathrm{~mm}, a=40 \mathrm{~mm}, s=0.25 \mathrm{~mm}$ были изначально заданы. Оптимальный расчетный показатель преломления $n_{c}=1.4076$ был вычислен в настоящей работе для температуры $25^{\circ} \mathrm{C}$. Данный концентратор обеспечивает попадание $90.92 \%$ „полезных“ фотонов, т.е. фотонов, поглощение которых приводит к появлению электроннодырочной пары, на ФЭП № 1 с фоточувствительной областью диаметром $1.7 \mathrm{~mm}$. Таким образом, общая кратность концентрирования получается $705^{x}$, а экономия площади ФЭП № 1, исходя из размера чипа $2 \times 2 \mathrm{~mm}$, составит 400 раз. В данном случае нет необходимости в обеспечении попадания на фоточувствительную область ФЭП № 1 всех „полезных“ фотонов, прошедших концентратор, т.е. 92.48\%. Это произойдет, если диаметр фоточувствительной области ФЭП № 1 составит $5.1 \mathrm{~mm}$. Но в этом случае кратность концентрирования снизится до $78^{x}$, а экономия площади чипов ФЭП № 1 до 55 раз.

Теоретическое исследование концентраторов солнечного излучения произведено при поддержке РФФИ грант № 14-08-00623. Экспериментальные исследования оптических свойств концентраторов солнечного излучения выполнены при поддержке гранта Российского научного фонда грант № 14-29-00178.

\section{Список литературы}

[1] Rumyantsev V.D., Davidyuk N.Yu., Ionova E.A., Larionov V.R., Malevskiy D.A., Pokrovskiy P.V., Sadchikov N.A., Andreev V.M. // Proceedings of the 5th International Conference on Solar. November 16-19. 2008. Palm Desert. CA USA. P. 13-3.

[2] Андреев В.М., Давидюк Н.Ю., Ионова Е.А., Покровский П.В., Румянщев В.Д., Садчиков Н.А. // ЖТФ. 2010. T. 80. Вып. 2. С. 118-125.

[3] Rumyantsev V.D. // Opt. Expr. 2010. Vol. 18. N S1. P. A24.

[4] ELASTOSIL® RT 604 A/B [Электронный pecypc]. Режим доступа: http://www.wacker.com/cms/en/products/ product/product.jsp?product=10463. Дата обращения: 01.02.2015.

[5] Shvarts M.Z., Andreev V.M., Gorohov V.S., Grilikhes V.A., Petrenko A.E., Soluyanov A.A., Timoshina N.H., Vlasova E.V., Zaharevich E.M. // Photovoltaic Specialists Conference, 2008. PVSC 2008. 33rd IEEE. 2008. P. 403 (on CD). 